\title{
Primary Pulmonary Lymphatic Flow Disorder Associated Plastic Bronchitis
}

\author{
Xiaoyan Zhang ${ }^{1}$, Jinrong $\mathrm{Liu}^{2}$, hui $\mathrm{xu}^{3}$, haiming $\mathrm{yang}^{3}$, and Shunying Zhao ${ }^{3}$ \\ ${ }^{1}$ Beijing Children's Hospital Capital Medical University \\ ${ }^{2}$ Beijing Children's Hospital Affiliated to Capital Medical University \\ ${ }^{3}$ Beijing Children's Hospital
}

January 10, 2022

\begin{abstract}
Lymphatic plastic bronchitis (PB) most commonly occurs in children with congenital heart disease as a result of secondary pulmonary lymphatic flow disorder (PLFD). However, PB caused by primary PLFD is rare. The clinical symptoms of lymphocytic PB are nonspecific; thus, the diagnosis may be long delayed, especially in the absence of chylothorax. We made a retrospective analysis of two children diagnosed with PB due to primary PLFD, in order to contribute to further understanding of these disorders. Patient 1, an eight-year-old boy, presented with chronic productive cough and expectorated milky-white mucous plugs accompanied by intermitted wheezing for one year. Patient 2, a nine-month-old girl, presented with episodes of acute respiratory distress with expectoration of milky-white bronchial casts for four months. There was no obvious evidence of infection in either child. Bilateral thickening of bronchovascular bundles and interlobular septal, as well as multiple patchy ground-glass opacities were seen on chest computed tomography (CT) in both patients. Lymphangioscintigraphy demonstrated pulmonary lymph reflux in both patients and slowed lymphatic drainage of the lower limbs in patient 1. Primary PLFD was considered for both patients, and a diagnosis of yellow nail syndrome was made in patient 1 . Both patients received lymphatic interventional treatment, but all experienced recurrence following the procedure.
\end{abstract}

\section{Primary Pulmonary Lymphatic Flow Disorder Associated Plastic Bronchitis}

To the Editor,

Plastic bronchitis (PB) is a rare disease characterized by the expectoration of rubbery, cohesive, and branched bronchial casts that obstruct the airway partially or totally. BP is associated with respiratory disorders such as bronchopulmonary infection, asthma, allergic bronchopulmonary aspergillosis (ABPA), and cystic fibrosis. Besides, BP commonly occurs in children with congenital heart disease, especially those with single ventricle physiology following the Fontan procedure. Elevation of central venous pressure after the procedure and secondary pulmonary lymphatic flow disorder (PLFD) leading to abnormal perfusion of the bronchial submucosa with lymph and formation of the airway cast ${ }^{1-2}$. However, PB caused by congenital lymphatic dysplasia and primary PLFD is rare, the clinical manifestation and severity vary among patients.

Patient 1 an eight-year-old boy admitted with a complain of chronic productive cough, expectorated milkywhite mucous plugs every 4-5 days (Figure. 1A), accompanied by intermitted wheezing for 1 year. Treatment of antibiotics, inhaled corticosteroids and brinchoidilaters fail to control the symptoms. His medical history revealed recurrent pneumonia as well as intermitted wheezing from two years of age, and he was diagnosed with asthma. His mother noticed the slow yellowing and thickening of the first toenail on his right foot about three years ago. The nail changes had been interpreted as onychomycosis and the first toenail had been removed before presentation to the hospital and a small and yellowish new toenail was observed (Figure. 1B). He was normally developed, there was no peripheral edema and the physical examination was unremarkable. 
The patient's white blood cell (WBC) count, eosinophil count, and C-reactive protein (CRP) were normal. The serum levels of aspergillus specific IgE, 1,3- $\beta$-glucan, and galactomannan were normal. Autoantibodies were all negative. Sputum cultures were positive for Candida albicans, while bacterial and mycobacterium cultures were negative. Fungal microscopic examination and culture of the first toenail were negative. Serial chest computed tomography $(\mathrm{CT})$ revealed migratory patchy ground-glass opacities and focal mild bronchiectasis with mucus impactions. The bronchial lavage fluid (BALF) was slightly milky-white and turbid, but no casts were observed. Fluid cultures showed no pathogens. BALF cell composition analysis was as follows: small lymphocytes $60 \%$, macrophages $20 \%$, exfoliated epithelial cells $20 \%$. The pulmonary function test showed mild obstructive ventilatory disturbance and the bronchodilation test was negative. An echocardiogram showed no abnormalities.

Suspicion of allergic bronchopulmonary mycosis (ABPM) was arised after the patient's admission. He was treated with fluconazole $(8 \mathrm{mg} / \mathrm{kg} . \mathrm{d})$ and prednisone $(1 \mathrm{mg} / \mathrm{kg} . \mathrm{d}$, gradually decreased to $0.5 \mathrm{mg} / \mathrm{kg} . \mathrm{d})$ for three weeks. However, his cough and expectoration of mucous plugs worsened. Repeated chest CT showed the progression of lung lesions with bilateral thickening of bronchovascular bundles, multiple patchy ground-glass opacities, and interlobular septal thickening, mainly in the middle-inferior lung, indicating pulmonary lymphatic stasis (Figure. 1C). Evaluation of the lymphatic system by lymphangioscintigray was performed subsequently and the results demonstrated slow lymphatic drainage in both lower limbs, abnormal distribution of the radiotracer in the bilateral hilar and lungs, suggesting pulmonary chylous reflux, as well as thoracic duct obstruction (Fig. 1D).

Based on the patient's clinical picture, lymphangioscintigraphy findings, and the yellow toenail on his right foot, the patient was finally diagnosed with yellow nail syndrome (YNS) and lymphatic PB. He was treated with a low-fat diet and percutaneous embolization of the thoracic duct was performed three weeks later. His respiratory symptoms were relieved. However, three months after the surgery, he experienced recurrence of bronchial cast expectoration occasionally. His parents refused further evaluation and treatment as he had developed no other symptoms.

Patient 2 a nine-month-old girl was referred to our department for episodes of acute respiratory distress with cough and expectoration of milky-white bronchial casts requiring bronchoscopy treatment for 4 months. During the course of the disease, there was no obvious fever. She had been admitted to the local intensive care unit and required mechanical ventilation two times because of severe bouts of dyspnea. Bronchoscopy demonstrated many milky-white casts blocking the airway (Figure. 2A); these were extracted by aspiration and her dyspnea and hypoxemia can be temporarily relieved. Chest CT demonstrated diffuse thickening of the interlobular septa and bronchovascular bundles, left partial atelectasis and consolidations, as well as enlargement of the mediastinum and hilar lymph nodes (Figure. 2B). Diagnoses of severe pneumonia and PB were made. However, despite vigorous therapy with antimicrobials (including antifungal drugs), intermittent systemic and inhaled glucocorticoids, and intravenous immunoglobulin, she continued to exhibit chronic expectoration of bronchial casts and episodes of acute respiratory distress requiring bronchoscopy to clear the airways about every two weeks. She had normal development and no history of trauma or surgery. Physical examination revealed tachypnea, a respiratory rate of 42 breaths/min, and oxygen saturation of $90 \%$ on room air. Chest auscultation showed bilateral moist rales and wheezing. The remainder of her examination was unremarkable.

The patient's WBC count, eosinophil count, hemoglobin, and CRP were normal. BALF cultures showed a small amount of Klebsiella pneumoniae. Tests for influenza virus, adenovirus, and mycoplasma detection were all negative. The echocardiogram showed no abnormalities. Abdominal ultrasound showed multiple cysts of the spleen. Lymphangioscintigraphy was performed subsequently and revealed thoracic duct obstruction, abnormal distribution of the radiotracer in the bilateral hilar and lungs, suggesting pulmonary chylous reflux (Figure. 2C).

Given her early age of onset, congenital lymphatic dysplasia was considered. Diagnostic pulmonary biopsy was not performed because of the risk of massive chyloptysis or refractory chylothorax ${ }^{3}$. Splenic cystic lesions were observed on abdominal ultrasound, suggesting extrathoracic involvement. The child was treated with 
high medium-chain triglycerides (MCT) milk powder, and oral rapamycin was administered $\left(0.8-1 \mathrm{mg} / \mathrm{m}^{2} . \mathrm{d}\right)$. Her symptoms had gradually improved and there were no further episodes of acute dyspnea for one month. Serum sirolimus levels were maintained at $8-13 \mathrm{ng} / \mathrm{mL}$ without any adverse reactions. Because the patient was still coughing up casts intermittently, percutaneous lymphatic intervention was performed (thoracic duct and right lymph duct outlet compression band loosening and stenotic adventitia stripping). Unfortunately, on postoperative day ten, she was readmitted with acute respiratory distress requiring emergent intubation. Bronchoscopy demonstrated that tenacious bronchial casts were obstructing the airways. After a series of bronchoscopies, her acute respiratory distress could not be alleviated, and thus, surgical ligation of the thoracic duct was performed. Her respiratory symptomsimproved. Currently, six months post-surgery, she is asymptomatic.

Here we described two cases of PB caused by primary PLFD with different clinical manifestations. Patient 1 was finally diagnosed as YNS. YNS is a rare disorder characterized by thickened yellow nails, primary lymphedema, and respiratory manifestations. The etiology of YNS remains unclear; however, a unifying lymphatic mechanism characterized by anatomical and/or functional lymphatic drainage abnormalities has been proposed ${ }^{4}$. The main respiratory system manifestations are bronchiectasis and pleural effusion, and about $20 \%$ of the pleural effusion cases are chylothorax ${ }^{4-5}$. In our patient 1 , there are no pleural effusion and lymphedema, the nail changes on the first toe had been interpreted as onychomycosis. thus, the consideration of chyloptysis and YNS are challenging, resulting in a long diagnosis delay. To our knowledge, this is the first report of YNS presenting as lymphatic PB which further supports a lymphatic mechanism of YNS. There was also diagnosis delay in patient 2 and unnecessary antimicrobial therapies were administered.

The chest CT findings, even if non-specific, were highly suggestive of PLFD. The typical findings include smooth thickening of the interlobular septa and bronchovascular bundles, patchy ground-glass opacities, mediastinal and hilar masses, as well as pleural effusions ${ }^{6}$. Our two cases both had features suggestive of PLFD. Pulmonary lymphatic flow disorder was demonstrated by lymphoscintigraphy in both children.

Due to the rarity of primary PLFD, there are no standardized treatment protocols. Recently, treatments with the mTOR inhibitor sirolimus have been shown to be effective in patients with lymphatic malformations, with good tolerability and few side effects ${ }^{7}$. In the current study, patient 2 received diet and sirolimus treatment only for one month and exhibited gradual improvement in her symptoms, thus the efficacy of these treatments could not be fully evaluated. It has been reported that percutaneous embolization of abnormal pulmonary lymphatic vessels in pediatric patients with a single-ventricle and in adult patients with PB results in alleviation of the symptoms ${ }^{2,8}$. However, the two children in the current study both had recurrence of casts after the lymphatic interventional procedure. Reasons for the recurrence might be associated with congenital lymphodysplasia, abnormal generation and proliferation of lymphatic vessels, as well as collateral development.

In conclusion, primary PLFD is a rare but important cause of PB in children. The clinical symptoms of lymphocytic PB are nonspecific; thus, the diagnosis may be long delayed, especially in the absence of chylothorax. Chest CT findings have highly suggestive significance for the diagnosis. Lymphatic interventional procedure is effective for short-term resolution of the symptoms, but it has a tendency to relapse.

Xiaoyan Zhang, MD

Jinrong Liu, MD*

Hui Xu, MD

Haiming Yang, MD,

Shunying Zhao, MD

Department of No. 2 Respiratory Medicine, Beijing Children's Hospital, Capital Medical University; National Center for Children's Health; China National Clinical Research Center of Respiratory Disease. Beijing, China

* Correspondence authors 
Jinrong Liu, Beijing Children's Hospital, No 56, Nan Li Shi Lu, Xicheng District, Beijing 100045, P.R. China (email:liujinrong2006@163.com ; Tel:010-59616314)

\section{Conflicts of interest}

All authors declare that there are no conflicts of interests.

\section{Authors' contributions}

XYZ was involved in diagnosis and treatment of both patients and drafted the initial manuscript. HX and HMY were involved in the diagnostic process of the patients. JRL and SYZ were responsible for the diagnosis and treatment of both patients, and revised the manuscript. All authors read and approved the final manuscript.

\section{References}

[1] Rubin BK. Plastic Bronchitis. Clin Chest Med. 2016;37(3):405-408.

[2] Dori Y, Keller MS, Rome JJ, et al. Percutaneous Lymphatic Embolization of Abnormal Pulmonary Lymphatic Flow as Treatment of Plastic Bronchitis in Patients with Congenital Heart Disease. Circulation. 2016; 133(12):1160-1170.

[3] Harnisch E, Sukhai R, Oudesluys-Murphy AM. Serious complications of pulmonary biopsy in a boy with chylopericardium and suspected pulmonary lymphangiomatosis. BMJ Case Rep 2010; 6; 2010: bcr08.2009.2206.

[4] Vignes S, Baran R. Yellow nail syndrome: a review. Orphanet J Rare Dis. 2017;12(1):42.

[5] Valdés L, Huggins JT, Gude F, et al. Characteristics of patients with yellow nail syndrome and pleural effusion. Respirology. 2014;19(7) :985-992.

[6] Swensen SJ, Hartman TE, Mayo JR, et al. Diffuse pulmonary lymphangiomatosis: CT findings. J Comput Assist Tomogr. 1995;19(3):348-52.

[7] Wiegand S, Wichmann G, Dietz A. Treatment of Lymphatic Malformations with the mTOR Inhibitor Sirolimus: A Systematic Review. Lymphat Res Biol. 2018;16(4):330-339.

[8] Itkin MG, McCormack FX, Dori Y. Diagnosis and Treatment of Lymphatic Plastic Bronchitis in Adults Using Advanced Lymphatic Imaging and Percutaneous Embolization. Ann Am Thorac Soc. 2016;13(10):1689-1696.

Figure legends

Figure 1. Patient 1. A, Expectorated milky-white mucous plugs. B, Yellow toenail on the right foot. C, Chest CT showing interlobular septal thickening and multiple patchy ground-glass opacities. D, Lymphoscintigraphy obtained 3 hours after injection reveals excessive accumulation of the radiotracer in the bilateral hilar and lungs.

Figure 2. Patient 2. A, Extracted bronchial cast with preservation of subsegmental airway divisions. B, Chest CT demonstrates diffuse thickening of the interlobular septa and bronchovascular bundles, left partial atelectasis and consolidations, enlargement of the mediastinum and hilar lymph nodes. C, Lymphoscintigraphy obtained 3 hours after injection reveals excessive accumulation of the radiotracer in the bilateral hilar and lungs. 

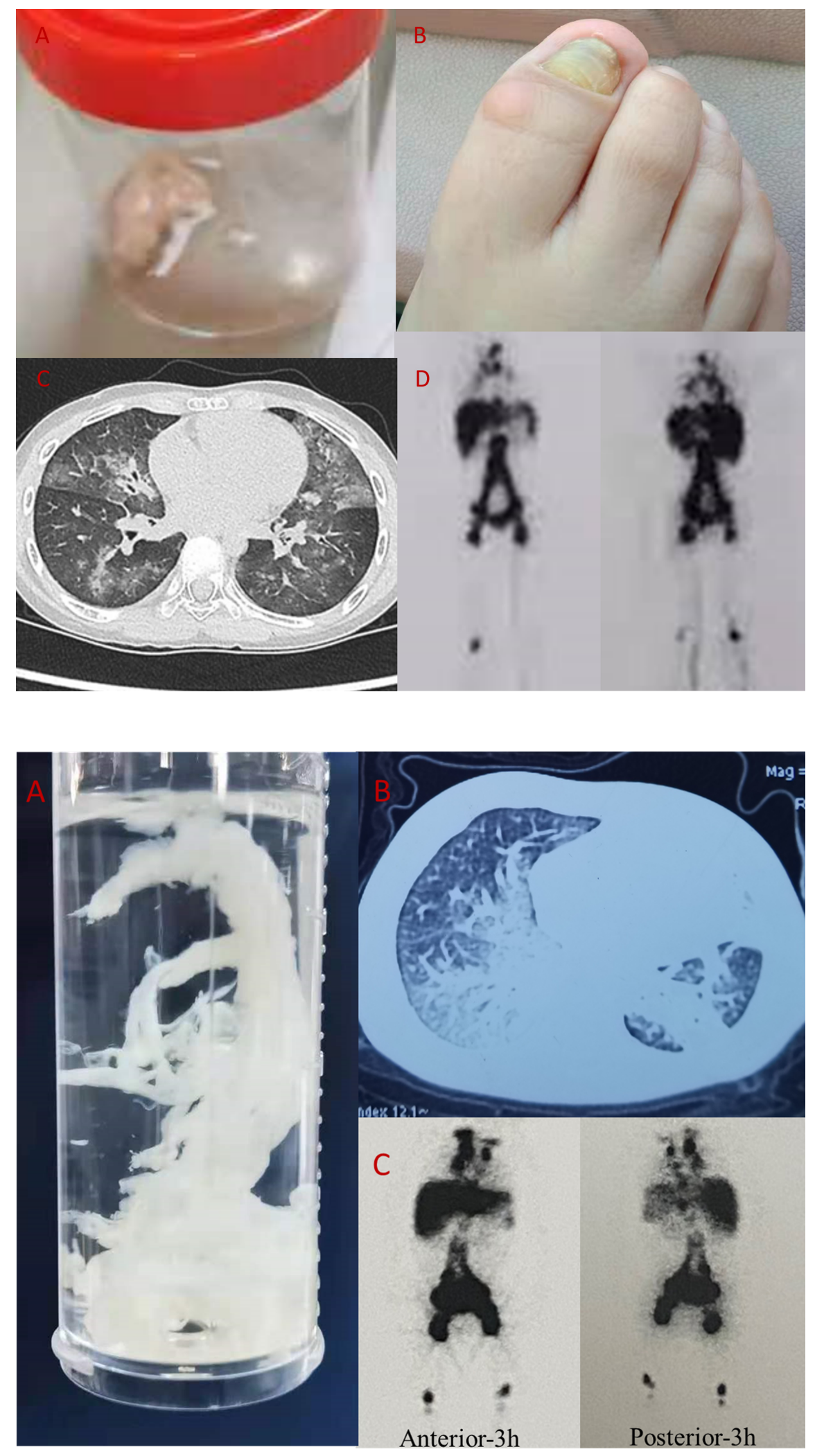